\title{
TODDLERS WITH ELEVATED AUTISM SYMPTOMS SHOW SLOWED HABITUATION TO FACES
}

\author{
Sara Jane Webb ${ }^{1,2,3}$, Emily J. H. Jones ${ }^{1,2}$, Kristen Merkle ${ }^{2}$, Jessica Namkung ${ }^{2}$, Karen $^{2}$ \\ Toth $^{1,4}$, Jessica Greenson ${ }^{2,3}$, Michael Murias ${ }^{1,2,3}$, and Geraldine Dawson 2,5,6,7 \\ ${ }^{1}$ Psychiatry and Behavioral Sciences, University of Washington, Seattle, WA, USA \\ ${ }^{2}$ University of Washington Autism Center, Seattle, WA, USA \\ ${ }^{3}$ Center on Human Development and Disability, University of Washington, Seattle, WA, USA \\ ${ }^{4}$ Seattle Children's, Seattle, WA, USA \\ ${ }^{5}$ Department of Psychology, University of Washington, Seattle, WA, USA \\ ${ }^{6}$ Department of Psychiatry, University of North Carolina, Chapel Hill, NC, USA \\ ${ }^{7}$ Autism Speaks, New York, NY, USA
}

\begin{abstract}
We explored social information processing and its relation to social and communicative symptoms in toddlers with Autism Spectrum Disorder (ASD) and their siblings. Toddlers with more severe symptoms of autism showed slower habituation to faces than comparison groups; slower face learning correlated with poorer social skills and lower verbal ability. Unaffected toddlers who were siblings of children with ASD also showed slower habituation to faces compared with toddlers without siblings with ASD. We conclude that slower rates of face learning may be an endophenotype of ASD and is associated with more severe symptoms among affected individuals.
\end{abstract}

\section{Keywords}

Habituation; Autism/ASD; Face processing; Toddlers; Broader phenotype

Processing information from faces is at the heart of learning about the social world, from reading others' emotions to understanding their focus of attention. Many researchers have thus been interested in exploring face processing in individuals diagnosed with an Autism Spectrum Disorder (ASD), who have marked impairments in social functioning in addition to difficulties with communication and the presence of restrictive or repetitive behaviors or interests. Such research has revealed that children and adults with ASD often perform poorly on behavioral tests of face processing and face recognition (for review, see Dawson, Webb, \& McPartland, 2005; Jemel, Mottron, \& Dawson, 2006; Sasson, 2006).

What is the origin of disruptions in face processing observed in ASD, and what are their consequences? Two hypotheses (that are notably not mutually exclusive) have been proposed (for review, see Dawson et al., 2005). First, face processing impairments in childhood or adulthood may be a consequence of other early-emerging social symptoms of ASD. The "social

(C) 2010 Psychology Press

Address correspondence to Sara Jane Webb, Box 357920, CHDD 314, Seattle WA 98195. sjwebb@u.washington.edu. 
motivation hypothesis" proposes that impairments in social motivation reduce attention to faces and other social stimuli, limiting the child's opportunities to develop expertise in social processing (Dawson et al., 2002; Grelotti, Gauthier, \& Schultz, 2002). Indeed, retrospective studies from home movies indicate that a decreased tendency to pay attention to faces is one of the earliest emerging social symptoms of ASD (Bernabei, Camaigni, \& Levi, 1998; Maestro et al., 2002, 2005; Mars, Mauk, \& Dowrick, 1998; Osterling \& Dawson, 1994; Osterling, Dawson, \& Munson, 2002; Zakian, Malvy, Desombre, Roux, \& Lenoir, 2000).

A second hypothesis posits that face processing is a primary area of impairment in ASD, caused either by direct impairments in the neural circuits underlying face recognition (e.g., van Kooten et al., 2008), or by more general low-level perceptual processing impairments that impact learning about perceptually homogenous classes of stimuli such as faces (for review, see Behrmann, Thomas, \& Humphreys, 2006). For example, a recent study found that children with ASD fail to develop perceptual expertise not only for faces but also for other stimulus classes that are difficult to discriminate (Scherf, Behrmann, Minshew, \& Luna, 2008). Other evidence suggests that there may be basic visual-processing impairments in infants at risk for ASD (McCleery, Allman, Carver, \& Dobkins, 2007), which the authors argue could contribute to later face-processing impairments.

If face-processing impairments emerge early in development, this may have a negative impact on the development of other early-emerging social and cognitive skills that rely heavily on processing information from human faces. For example, joint attention is enhanced when an adult's eye direction is visible (Brooks \& Meltzoff, 2002, 2005), recognizing emotion involves detecting facial expressions (Adolphs, 2002; Adolphs \& Tranel, 2003), language processing is facilitated by analysis of mouth movements (Kuhl, Tsao, \& Liu, 2003; McGurk \& McDonald, 1976), and word learning is facilitated by gaze following (Baldwin, 1993; Brooks \& Meltzoff, 2005; Hollich, Hirsh-Pasek, \& Golinkoff, 2000; Houston-Price, Plunkett, \& Duffy, 2006; Moore, Angelopoulos, \& Bennett, 1999; Tomasello \& Barton, 1994). Early face-processing impairments may thus represent a risk factor for the development or exacerbation of some of the social and communicative symptoms of ASD, and examining this relation is central to evaluating the importance of including face-processing skills in an early intervention program.

Understanding the origins and consequences of face-processing impairments thus requires an integrated knowledge of the early development of face processing, and social and communication skills, in individuals with ASD. However, few studies have examined face processing prior to age 3 years, and existing studies draw mixed conclusions (Chawarska \& Volkmar, 2007; Jones, Carr, \& Klin, 2008). While Jones et al. found marked differences in how 2-year-olds with ASD or general developmental delay scanned facial features during a period of live interaction, Chawarska and Volkmar (2007) found no differences in face memory between 1- to 3-year-olds with ASD or more general developmental delays. Further, while scanning patterns in the former study were related to social skills, face memory in the latter study was not correlated with socialization or interaction abilities.

A further way to address the developmental origins of face-processing impairments in ASD is to examine face processing in first-degree relatives of individuals with ASD. Heritability rates indicate that ASD likely has a strong genetic component, but inheritance patterns are complex (e.g., Bailey et al., 1995; Steffenburg et al., 1989; Freitag, 2007; Gupta \& State, 2007; though see Skuse, 2007). Characterizing the relation between genotype and phenotype in disorders with complex inheritance patterns is facilitated by the identification of "endophenotypes," heritable traits that lie between genotype and the clinical phenotype that leads to a diagnostic classification (Gottesman \& Gould, 2003). One of the five criteria for identifying endophenotypes in disorders like ASD is the presence of the endophenotype in unaffected family members at a higher rate than in the general population (Gottesman \& Gould, 2003; 
Leboyer et al., 1998). Indeed, parents of individuals with ASD may exhibit qualitatively similar but milder deficits in face processing to those seen in individuals with ASD, suggesting that face-processing impairments may be a heritable risk factor or endophenotype of ASD (Adolphs, Spezio, Parlier, \& Piven, 2008; Dawson et al., 2005).

Assessing face processing in the early development of younger siblings of children with ASD also enables this endophenotype criterion to be assessed. Intriguingly, recent prospective studies have found that at 6 months, some siblings of children with ASD show atypical patterns of visual fixation and attention-shifting during face-to-face episodes with their mother (Ibanez, Messinger, Newell, Lambert, \& Sheskin, 2008; Merin, Young, Ozonoff, \& Rogers, 2007), suggesting that some patterns of behavior seen in toddlers with ASD (Jones et al., 2008) may be shared with their siblings. However, it is presently unclear whether these effects were driven by infants who themselves went on to develop ASD. Thus, the early development of face processing in neurotypical first-degree relatives of individuals with ASD remains an important topic for further investigation.

\section{Assessing Face-Processing Abilities in ASD and Young Children}

Characterizing face processing in young children with ASD is thus both theoretically and empirically important. However, toddlers and young children with ASD often have significant delays that can present challenges for behavioral testing. One strategy for assessing young children with significant developmental delays is to incorporate methodologies that have proven to be successful in studying cognition in infants, such as the habituation paradigm (for review, see Colombo \& Mitchell, 2008).

Habituation is defined as a decrease in looking time over multiple repetitions of a stimulus (Slater \& Bremner, 1989). Decreases in looking time are thought to reflect the infant becoming "bored" with a stimulus of which he or she has formed a mental representation. The presentation of a new stimulus may reengage the infant's interest, indicating that decreases in looking during habituation were stimulus specific. In an infant-controlled habituation task, the amount of familiarization time each infant receives is controlled by his or her habituation function. When the infant's looking drops below a certain level (typically defined as a 50\% decrease in looking), he or she is considered to have habituated to the stimulus. Time taken to reach the habituation criterion can be used as a proxy measure of learning rate. During the test phase, the infant is sequentially presented with the familiarization stimulus and a novel stimulus. Greater looking to the novel stimulus relative to the familiar stimulus is typically interpreted to reflect memory for the familiar stimulus. By varying the delay between the test phase and the learning phase, researchers can examine the duration over which memory for the familiarization stimulus persists.

Research with habituation paradigms (or related visual attention procedures) has provided insight into the development of face processing in typically developing infants and young children. For example, Fagan (1974) showed that 6-month-old infants learn about faces faster than they learn about line drawings of objects (with a novelty preference requiring a familiarization time of 22 seconds for faces, and 35 seconds for line drawings). Pascalis, de Haan, Nelson, and de Schonen (1998) showed that 6-month-old infants could learn and remember a briefly presented face for up to 24 hours, and by 12 months infants can recognize a novel face across a change of room (Jones, Pascalis, Eacott \& Herbert, in press). Habituation procedures can also provide important information regarding individual differences in learning and memory for faces. For example, habituation time in infancy is a significant predictor of later IQ (for meta-analysis see Bornstein \& Sigman, 1987; McCall \& Carriger, 1993) and memory abilities (Rose, Feldman, \& Wallace, 1992; Thompson, Fagan, \& Fulker, 1991; though see Fagan, 1984). Further, the length of time infants take to habituate to watching an adult 
paying attention to an object is a significant predictor of later social skills (Wellman, Phillips, Dunphy-Lelii, \& LaLonde, 2004), suggesting that measuring habituation to social stimuli in infancy may provide valuable insight into social development.

\section{Current Study}

The current study examined face processing and its relation to social and communication skills in toddlers with ASD, toddlers with general developmental delay (DD), typically developing toddlers, and toddlers with siblings who have ASD. To measure face processing, we used an infant-controlled habituation procedure to assess learning and memory for faces and objects (houses). Houses were chosen as a control stimulus because they share properties of symmetry and configuration with faces. Recent analyses of the underlying factors influencing performance in infant studies indicate that different factors account for habituation and recognition memory, confirming the importance of providing separate estimates of these abilities (Rose, Feldman, \& Jankowski, 2004, 2005). To measure other aspects of development (including social and communicative abilities), we used information from the Mullen Scales of Early Learning (Mullen, 1997), the Vineland Adaptive Behavior Scales (Sparrow et al., 1984), and the Autism Diagnostic Observation Scale (ADOS; Lord, Rutter, DiLavore, \& Risi, 1999), which were administered to the children as part of a larger study.

This project posed the following specific questions:

Is face processing impaired in toddlers with ASD, and are face-processing impairments related to general developmental delays or are they specifically associated with social impairments? If toddlers with ASD have face-processing impairments, we would expect longer habituation times to faces compared to houses. If toddlers with ASD have general information-processing deficits, we would expect longer habituation times to both stimuli compared to the other groups. If faceprocessing impairments are either a risk factor or a consequence of other social impairments, we would expect there to be a relation between social skills and faceprocessing impairments in toddlers with ASD. Alternatively, if disrupted face processing is related to general developmental delays, we would expect a relation between chronological age, nonverbal skills, and face processing in toddlers with ASD and toddlers with developmental delay.

Is face memory impaired in toddlers with ASD? To assess memory, novelty preferences were tested after a 5-second delay, or a 5-minute delay. If toddlers with ASD have general impairments in learning or memory, performance may be impaired at both the 5-second and 5-minute delay to both face and house stimuli. Alternatively, if deficits are specific to memory, performance should be impaired at the 5-minute delay only.

Areface-processing impairments present in siblings of children with ASD? To address this question, the same procedures were used with groups of toddler siblings of children with ASD. This included toddler siblings of children with ASD who were typically developing, and toddler siblings of children with ASD who were experiencing developmental delays in motor, social, communication, or cognitive abilities. If face processing and memory are specific areas of impairment in ASD, or a consequence of other social impairments, we would expect toddlers with ASD to perform worse than toddler siblings with typical development or developmental delays. However, if face-processing impairments are an endophenotype of ASD (Dawson et al., 2005), then we would expect that both toddlers with ASD and toddler siblings would show impaired performance relative to comparison groups. 


\section{METHODS}

\section{Participants}

Four groups of children aged between 18 and 30 months participated in the study: typically developing toddlers with no family history of ASD (TD), toddlers with ASD (ASD), toddlers with developmental delay or language delay with no family history of ASD (DD), and toddlers with a sibling with ASD who did not themselves have an ASD diagnosis (SIB). Participants were recruited from clinics, hospitals, local advocacy groups, and the university Infant and Child Subject Pool.

All children participated in a larger study of early development of autism, which included standardized diagnostic, cognitive, adaptive, and language assessments as well as an experimental battery including social and cognitive tasks. Children were evaluated using the Vineland Adaptive Behaviors Scales-Expanded (Sparrow et al., 1984), the Autism Diagnostic Interview-Revised (ADI-R; Lord, Rutter, \& LeCouteur, 1994), the Autism Diagnostic Observation Schedule (ADOS; Lord, Rutter, DiLavore, \& Risi, 1999), the Preschool Language Scale, 4th Edition (Zimmerman, Steiner, \& Pond, 2002), and the judgment of a team of highly experienced clinicians (GD, JG, KT) based on Diagnostic and Statistical Manual of Mental Disorders, 4th Edition criteria (DSM-IV; American Psychiatric Association, 1994). The Mullen Scales of Early Learning (MSEL) were used to assess cognitive and motor function and included the domains of fine motor, gross motor, visual reception, receptive language, and expressive language. Scores were converted into developmental quotients (DQ) by dividing age-equivalent scores by chronological age and multiplying by 100 (e.g., Lord et al., 2006; Munson et al., 2008). Of note, whilst the use of age-equivalent scores in the MSEL has been recommended for the assessment of young children with ASD because other metrics produce significant floor effects (Akshoomoff, 2006), one challenge to their interpretation is that the same age-equivalent score can be generated by different skill profiles. Information about age, gender, nonverbal and verbal DQ, and autism symptoms are presented in Table 1. All children were medication free and had no history of head trauma, seizure disorder, chronic medical condition, or a genetic syndrome associated with autism. Group assignments were based on diagnostic assessments conducted at the time of habituation testing. However, some of the children in the DD (81\%) and ASD (68\%) groups participated in follow-up assessments (using the MSEL, VABS, ADOS, and ADI-R) 1 to 2 years after their participation in the habituation experiment. Children who changed diagnostic category were further excluded (reported below).

The ASD group ( $n=69,17$ female) met criteria for autism or autism spectrum disorder on the ADOS-Module 1 social and communication domains, within 2 points of autism on the ADI$\mathrm{R}$, and autistic disorder or pervasive developmental disorder not otherwise specified based on $D S M-I V$ criteria. Ten toddlers in the ASD group had older siblings with ASD. For analysis purposes, a further subdivision was made between groups of children in the study based on their scores on diagnostic measures. Specifically, the ASD group was split into a higher symptom and a lower symptom group, based on a median split of their ADOS-G (module 1) social and communication algorithm scores. This resulted in an ASD-High Symptom (ASDHS) group with ADOS scores of 17 and above ( $n=34,5$ female), and an ASD-Low Symptom group (ASD-LS) with ADOS scores of less than 17 ( $n=35,12$ female). This was done to attempt to capture the variability in symptom number and severity that exists within toddlers who receive a diagnosis of ASD.

We included three comparison groups. Children were included in the typical developing group (TD; $n=31$ ) if they scored above 85 on the Mullen composite scores and on the Vineland Adaptive Behavior Scale (VABS) composite score and subdomain scores. 
Toddlers were initially included in the developmental delay (DD) group $(n=22)$ if they had standard scores below 85 on the composite score and below 35 on at least two subdomains of the Mullen. These participants also had standard scores of below 85 on at least two of the VABS subdomains (communication, daily living, socialization, or motor skills); this included 17 DD subjects who were considered to have "idiopathic" delays, and 5 with delays that resulted from other medical conditions ( 3 with Down's Syndrome, 1 with Williams Syndrome, and 1 with premature birth). To increase sample size, a further 5 participants with specific language delay were included in the DD group, resulting in a final sample of 27. (Specific language delay was defined as a standard score below 35 on the Expressive Language domain of the Mullen, and a standard score below 80 on the Expressive Communication domain of the Preschool Language Scale, 4th Edition.)

Toddlers were included in the sibling group (SIB; $n=48$ ) if they had an older sibling who met criteria for autism spectrum disorder on the ADI-R, ADOS, and based on clinical judgment using $D S M-I V$ criteria. Because younger siblings of children with autism may display a range of abilities, we further subdivided this group. The DD-SIB group included 19 children with impairments in expressive language ( $n=4,0$ female) or receptive language ( $n=10,4$ female) or both ( $n=2,2$ female), defined as a standard score below 35 on the relevant domain of the Mullen; or social-cognitive delay defined as ADOS social scores of greater than 6 but no formal diagnosis of ASD ( $n=3,2$ female). The TD-SIB group included 29 ( 12 female) children who scored within the average range on standardized assessment measures.

Final sample-Data were excluded for an additional 12 toddlers due to noncompliance with the testing procedure $(n=2)$, desire to withdraw from the wider study $(n=1)$, known change in diagnostic profile $(n=3)$, or inconclusive diagnostic profiles $(n=6)$. The final sample included 66 toddlers with ASD (33 ASD-HS, 33 ASD-LS), 29 typically developing toddlers (TD), 20 toddlers with developmental delay (DD), and 48 toddlers with siblings with ASD (19 DD-SIB; 29 TD-SIB). Descriptive information of chronological age, sex, ADOS total score, and DQ for the six groups of toddlers is presented in Table 1.

\section{Stimuli}

Stimuli were grayscale photographs of female faces and houses, measuring $16 \mathrm{~cm}$ by $18 \mathrm{~cm}$. Houses were chosen to be symmetrical and forward facing. Four pairs of stimuli were used in each condition (faces or houses) to ensure findings were not stimulus specific. Stimuli were counterbalanced across participants, such that approximately equal numbers of participants in each group viewed each house and face pair. Pairs of stimuli were judged to be approximately matched on attractiveness, luminance, and complexity. Examples of stimuli are presented in Figure 1.

\section{Habituation Procedure}

Children participated in four habituation experiments, in a two stimulus (houses and faces) by two delay ( 5 seconds vs. 5 minutes) repeated measures design. Testing was conducted on two different days. At each visit, one test involving faces and one test involving houses were presented. One test at each visit was conducted with the short delay (5 seconds), and one was conducted with the long delay ( 5 minutes). Order of testing for both stimulus and delay was counterbalanced within these restrictions (i.e., Day 1 Face - Long delay and House - Short delay; Day 2 House - Long delay and Face - Short delay).

When possible, the child was seated in a high chair, with a behavioral assistant sitting by his or her side. If this was not possible, the child was seated on the parent's lap. If the high chair was not used, the parent was blindfolded or was asked to close their eyes during the experiment. 
Parents were asked to refrain from providing verbal or nonverbal cues during the testing procedure.

During the habituation phase, two experimenters (who were aware of the diagnostic status of the child) measured looking time by pressing a button while the child visually fixated the stimulus. The stimulus was removed from the screen if the child looked away for more than one second. When this occurred, an "attention getter" (a flashing colored square accompanied by a chirping noise) was used to regain the child's attention to the screen. When the child attended to the screen for longer than one second, the stimulus was re-presented. We used a reorienter to maximize participant retention and to reduce the potential effect of differences in endogenous orienting on look spacing and habituation times (Rankin et al., 2009). This procedure has been used in many previous studies (e.g., Bornstein \& Benasich, 1986; Casasola \& Bhagwat, 2007; Lewkowicz, 2004: Saffran, Pollak, Seibel, \& Shkolnik, 2007; Soska \& Johnson, 2008; Strauss \& Curtis, 1981) and is included in a leading computer program used to implement habituation protocols (Cohen, Atkinson, \& Chaput, 2004).

Habituation was defined as having been met when the average of two consecutive looks fell below 50\% of the average of the child's longest two looks, requiring a minimum of three looks. Of note, a look could contribute to both the average of the longest looks, and the two consecutive looks used to define the habituation point. A "look" was defined as visual fixation for greater than one second. These calculations were implemented by a computer running inhouse software. The two longest looks were chosen as the criterion because, although an averaged habituation function decreases monotonically, around $40 \%$ of individual infants actually produce their peak looks later in the habituation function (Bornstein \& Benasich, 1986; Colombo \& Mitchell, 1990; Colombo, Mitchell, O'Brien, \& Horowitz, 1987).

The habituation phase was followed by a delay phase of either five seconds or five minutes. During the delay phase, the child was not shown any stimulus. Children remained in the testing room for the duration of the delay period. In the testing phase, the familiar stimulus and a previously unseen stimulus of the same stimulus category were presented in random order. The stimuli were presented for the duration of one look.

Reliability of testing was first assessed by calculating the Pearson correlation coefficient between the coding times of the first and second experimenter for all infants. If the correlation was less than $r=.8$, the video recording was re-coded offline by trained coders. If the correlation remained less than $r=.8$, or if there were significant disagreements in the number of looks, the data were dropped from further analysis $(n=5$ of 608). Data from 39 habituation and test periods were dropped for child behavior (e.g., fussiness; $n=27$ ); experimenter error $(n=7)$; equipment malfunction $(n=2)$; maternal interference $(n=2)$. In addition, data from an additional 34 test periods were excluded for the same reasons. Thus, there were 564 valid habituation sessions and 530 valid test sessions included in the final analyses. (This exclusion rate is very favorable in comparison to standardized measures used with mixed groups that may have a dropout rate of over 50\% [Klin et al., 1999].) The IntraClass Correlation Coefficient was calculated for 225 habituation and test periods; the mean ICC was $0.97(S D=0.1)$.

\section{Analysis}

Demographic characteristics of each group, and their performance on the standardized measures, are presented in Table 1. Univariate ANOVAs with post-hoc Bonferroni-corrected $t$-tests were conducted to explore group differences in age and standardized assessments. These revealed a main effect of group on every measure (see Table 1). Post-hoc tests with an alpha level of .05 revealed that, in general, the ASD groups significantly differed from the DD and TD groups on every measure. The TD group and the TD-SIB group did not significantly differ from each other. The DD and DD-SIB groups did not differ from each other but differed from 
the ASD and TD groups. Given the differences in age and nonverbal ability between the samples, group analyses were run with and without age and nonverbal ability as covariates. Since the ADI-R, Vineland, and verbal ability measures may be used in the diagnosis of ASD, differences between groups are expected and do not indicate that these measures should be partialled out of further analysis (Hobson, 1991;Jarrold \& Brock, 2004).

Habituation analysis strategy-As children participated in two face habituation experiments and two house habituation experiments, data were averaged across the two experiments in each condition to provide a more stable characterization of individual differences (Colombo, Mitchell, \& Horowitz, 1988; Rose, Feldman, \& Wallace, 1988). If a child had only one valid data point for either the house or the face condition, this data point alone was included in the analysis. This enabled us to maximize the number of children included in the final analysis. Preliminary tests revealed no significant effects of the number of data points included in the analysis for each child (all $p>.1$ ), and so this variable was excluded from further analyses. To be included in the analysis, children had to have at least one valid habituation to a face stimulus and one valid habituation to a house stimulus. This excluded data from a further 6 children who did not have valid data in both conditions (ASDLS $n=0$; ASD-HS $n=2$; DD $n=1$; TD $n=2$; DD-SIB $n=1$; TD-SIB $n=0$ ). Finally, for each analysis, values lying more than three standard deviations outside the mean of each habituation variable were excluded (range: 0 to 6 excluded per analysis).

The habituation phase of the experiment yielded several related measures: total time to habituate, peak look during habituation, number of looks to criterion, and mean look during habituation. Descriptive statistics for all measures are provided in Tables 2 and 3. Peak look, mean look, and time to habituate provide strongly interrelated characterizations of visual attention during the habituation phase. Consonant with this, the pattern of results across these three variables was highly conserved. As seen in Table 3, the ASD-HS group took longer to habituate to faces, had a longer peak look during habituation to faces and had a longer mean look during habituation to faces in comparison to the other groups. Detailed analyses are presented for time to habituate and total look number. Final analyses used a repeated measures analysis with group as a between-subjects factor and stimulus (face or house) as the repeated measures factor. Follow-up analyses were used to interpret significant interactions.

\section{RESULTS}

\section{Habituation Phase - Number of Looks}

A repeated measures ANOVA on number of looks by stimulus (face or house) and group (ASDLS, ASD-HS, DD, TD, TD-SIB, and DD-SIB) revealed no main effect of group, $F(5,147)=$ $1.9, p=.1$, no main effect of stimulus, $F(5,147)=0.1, p=.8$, and no stimulus by group interaction, $F(1,147)=1.1, p=.4$. Thus, the number of looks taken to reach the habituation criterion was broadly similar across groups and procedures.

\section{Habituation Phase - Time to Habituate}

A repeated measures ANOVA on time to habituate by stimulus (face or house) and group (ASD-LS, ASD-HS, DD, TD, TD-SIB, and DD-SIB) revealed a main effect of stimulus, $F(1$, $142)=4.0, p=.047$, and a main effect of group, $F(5,142)=7.0, p<.001$, qualified by an interaction between group and stimulus, $F(5,142)=5.0, p<.001$.

To interpret the interaction between stimulus and group, we conducted a series of planned repeated measures analyses for time to habituate to faces versus houses in each group separately. Children in the ASD-HS group had a significantly different pattern of habituation than the other groups. Specifically, children in the ASD-HS group looked significantly longer 
during face habituation than house habituation, $F(1,30)=13.5, p=.001$, whereas there were no significant differences in speed of habituation to faces versus houses for all other groups, ASD-LS: $F(1,31)=0.2, p=.7$; DD: $F(1,16)=0.01, p=.9$; TD: $F(1,23)=0.01, p=.9$; DDSIB: $F(1,17)=0.1, p=.8$; TD-SIB: $F(1,25)=0.001, p=.97$. Finally, a univariate ANOVA of time to habituate to faces revealed a main effect of group, $F(5,147)=10.4, p<.001$, and post-hoc Tukey tests controlled for multiple comparisons indicated that toddlers in the ASDHS group took significantly longer to habituate to faces than any other group of toddlers $(p<$. 001 ). An analogous analysis on time to habituate to houses revealed no group differences, $F$ $(5,143)=1.6, p=.2$. In summary, toddlers with more severe symptoms of autism took significantly longer to habituate to faces than houses and took significantly longer to habituate to faces than any other group (see Figure 2).

\section{Habituation Phase - Relation to Phenotype}

Finally, to test the prediction that longer looking to faces is a feature of the broader ASD phenotype, univariate ANOVAs were conducted on time to habituate to faces or houses by sibling group (with or without a sibling with ASD) in typically developing toddlers and toddlers with developmental delays. Results revealed that TD-SIB group took significantly longer to habituate to faces than the TD group, $F(1,50)=6.3, p=.015$, while there was no significant difference in habituation time to houses, $F(1,50)=2.0, p=.16$. In contrast, there were no significant differences between time to habituate to faces or houses between the DD and DDSIB groups, $F(1,34)=0.13, p=.72$. Notably, these effects were not seen in the analyses above, where post-hoc tests were conducted controlling for multiple comparisons, indicating that the difference between the TD and TD-SIB groups was relatively small. It is also important to note (see Figure 2) that the TD-SIB group did not show slowed face processing relative to house processing; rather, the TD group was the only group to show slightly faster face than house processing, a difference that was not observed in the TD-SIB group. Further, it is clear from Figure 2 that the DD, DD-SIB, ASD-LS, and TD-SIB groups took similar lengths of time to habituate to the face (and house) stimuli. Thus, toddlers with developmental delay or a sibling with ASD did not show the same speeded habituation to a face as typically developing toddlers with no risk factors.

\section{Test - Novelty Score}

In order to establish whether each group showed evidence of recognition memory for the familiar stimulus at each delay, novelty scores were computed: look time to novel was divided by the sum of look time to novel and look time to familiar (Table 4). A novelty score greater than 0.5 indicates that the group looked proportionally more at the novel stimulus than at the familiarized stimulus during the test session. These were compared to the theoretical chance value of 0.5 with a series of one-sample $t$-tests.

For the 5-second delay condition, this analysis revealed that only the ASD-HS group and the DD-SIB group showed significant novelty preferences with face stimuli, $t(27)=2.2, p=.04$; $t(14)=3.2, p=.006$, while only the TD-SIB group showed significant novelty preferences with house stimuli, $t(24)=2.4, p=.03$. The TD-SIB group showed a trend towards significance for the face stimuli, $t(23)=1.89, p=.07$, and the DD-SIB group showed a trend towards significance for the house stimuli, $t(16)=1.9, p=.08$. All other groups did not show a significant preference (all $t<1.5, p>.2$ ).

For the 5-minute delay condition, this analysis revealed that only the DD-SIB group showed significant novelty preferences with face stimuli, $t(16)=4.4, p=.001$, and only the TD group showed significant novelty preferences with house stimuli, $t(20)=3.3, p=.004$. All other groups did not show a significant preference (all $t<1.5, p>.2$ ). 
Lastly, a repeated measures analysis with stimulus (face versus house) and delay (5 seconds versus 5 minutes) was conducted including the children who provided valid data for all four measures. This revealed no main effect of group, $F(5,86)=1.84, p=.11$, no main effect of stimulus, $F(1,86)<0.001, p=.99$, no main effect of delay, $F(1,86)=0.58, p=.13$, and no significant interactions (all $F \mathrm{~s}<1.5, p>.3$ ). Thus, the novelty preferences did not reveal significant differences in memory between faces and houses or between the diagnostic groups.

\section{Predictors of Habituation}

As time to habituate to faces differed between groups, a series of regression analyses was run to establish the relation between performance on the face habituation tasks and scores on the standardized developmental and diagnostic measures. The ASD-HS and ASD-LS groups differed on multiple variables (including ADOS scores, age, nonverbal DQ, verbal DQ, and Vineland Socialization and Communication scores), and it was thus important to explore which of these variables might be related to prolonged habituation times to faces in the ASD group. We also wanted to explore whether the same relations were observed in the DD and TD groups.

In order to ensure that correlations were not negatively influenced by the presence of clusters of children for whom standardized measures bore a different relation to each other, a cluster analysis was first run to establish the optimum groupings for the regression analyses. A twostep cluster analysis including scores from the ADOS, the Vineland, the Mullen, and group (TD, TD-SIB, DD, DD-SIB, ASD-LS, and ASD-HS) revealed three clusters (Bayesian Information Criterion $=559.9$ ) . Table 5 shows the proportion of children classified in the six groups who fell into the three clusters. This analysis suggested that the best way to divide up the initial six groups for the regression analyses was by their diagnostic status (ASD, DD, or TD), rather than by the groups in which they were initially recruited to the study (Siblings, $\mathrm{DD}, \mathrm{TD}$, or ASD), or by examining predictors in the group as a whole. Notably, ADI-R scores were not entered into the analysis, as this measure was not available for the TD group. This likely accounts for the poorer classification of the children with ASD, since the ADI is an important part of the diagnostic battery for this group. Nonetheless, it is important to note that some of the low-symptom ASD children showed a similar profile to children with more general developmental delay.

To assess what variables were related to differences in looking time, the children were split into three broad groups based on their diagnostic status: children with a diagnosis of ASD $\left(\mathrm{ASD}_{\mathrm{all}}=\mathrm{ASD}-\mathrm{HS}+\mathrm{ASD}-\mathrm{LS}\right)$, children with some developmental delays $\left(\mathrm{DD}_{\text {all }}=\mathrm{DD}+\mathrm{DD}-\right.$ $\mathrm{SIB})$, and typically developing children $\left(\mathrm{TD}_{\mathrm{all}}=\mathrm{TD}+\mathrm{TD}-\mathrm{SIB}\right)$. These analyses were carried out with total time to habituate to faces as the dependent variable. Two analyses were used:

(a) the corresponding measure for the house experiment, age, sibling status, nonverbal DQ and ADOS scores were included as predictors; (b) the corresponding measure for the house experiment, age, sibling status, verbal DQ, Vineland Socialization standard score, and Vineland Communication standard score were entered as predictors. Analyses were split to prevent excessive collinearity between predictors.

Overall, the two models predicted $26 \%$ and $24 \%$ of the variance in the $\mathrm{ASD}_{\text {all }}$ group, Model 1: $F(5,60)=3.84, p=.005$; Model 2: $F(6,53)=2.68, p=.024$, and $29 \%$ and $26 \%$ of the variance in the $\mathrm{TD}_{\text {all }}$ group, Model 1: $F(5,44)=3.0, p=.022$; Model 2: $F(6,44)=3.61, p=$. 006 . The models only predicted a nonsignificant $16 \%$ and $14 \%$ of the variance in the $\mathrm{DD}_{\text {all }}$ group, Model 1: $F(5,31)=0.95, p=.47$; Model 2: $F(6,31)=0.53, p=.66$.

For the $\mathrm{TD}_{\text {all }}$ group, time to habituate to houses predicted time to habituate to faces $(B=0.37$, $t=2.4, p=.021$ ). Variation in general object-processing mechanisms predicts a significant amount of variation in face-processing mechanisms in this group. Total time to habituate to 
faces was also predicted by sibling status $(B=0.51, t=3.27, p=.002)$, such that the TD-SIB group took longer to habituate to faces than the TD group.

For the $\mathrm{ASD}_{\text {all }}$ group, time to habituate to houses $(B=0.39, t=3.38, p=.001)$, ADOS total score $(B=0.25, t=2.03, p=.048)$, and verbal DQ $(B=-0.34, t=-2.05, p=.046)$ predicted time to habituate to faces. As in the $\mathrm{TD}_{\text {all }}$ group, variation in general object-processing mechanisms predicted a significant amount of variation in face-processing mechanisms in this group. However, ADOS scores and verbal DQ contributed significantly to the remainder of the variance in the face looking measures, such that children with higher symptom levels and lower verbal DQs took longer to habituate to face stimuli.

In the $\mathrm{DD}_{\text {all }}$ group, there were no significant predictors of time to habituate to faces (all $t \mathrm{~s}<$ $2, p>.1$ ). Thus, looking time measures to houses did not consistently predict looking time to faces in the $\mathrm{DD}_{\text {all }}$ group.

\section{DISCUSSION}

The present study examined learning and memory for social and nonsocial stimuli in toddlers with ASD, toddlers with more general developmental delays, typically developing toddlers, and toddlers with siblings who have ASD. Learning was measured by testing habituation speed to a face or a house stimulus. Memory was measured by examining dishabituation to a novel within-category stimulus (relative to the habituated stimulus) after a 5-second or 5-minute delay. Finally, regression analyses were used to explore relations between face learning and other domains such as house learning, age, verbal DQ, nonverbal DQ, social skills, and communication skills. The next paragraphs address the three questions posed in the introduction. Answering these questions provides insight into the developmental origins of face-processing impairments in ASD.

\section{Is Face Processing Impaired in Toddlers with ASD?}

In the present study, the ASD-HD group took significantly longer to habituate to faces than houses and took significantly longer to habituate to faces than children in the ASD-LS, TD, TD-SIB, DD, and DD-SIB groups. Regression and ANCOVA analyses revealed that these findings could not be attributed to age or nonverbal DQ differences between groups or to differences in the number of looks to the stimulus. Rather, verbal ability and social and communication scores were significantly predictive of habituation time to faces. Thus, toddlers with ASD who were experiencing more severe social and communication symptoms showed a domain-specific slowing in face processing that was not observed in toddlers with more general developmental delays.

Previous work also indicates that face-processing impairments are unlikely to be a universal feature of ASD, with substantial individual differences between participants being observed (e.g., Barton, Cherkasova, Hefter, Cox, O'Connor, \& Manoach, 2004; Behrmann et al., 2006; Boucher, Lewis, \& Collis, 1998). The present findings suggest that in toddlerhood social and communication symptom level may be one factor that influences the degree of slowed face processing exhibited by a group of individuals with ASD. As well, Jones et al. (2008) found that decreased looking to the eyes of a dynamic stranger was correlated with higher ADOS scores in 2-year-olds with ASD. Further, Klin et al. (1999) found that while 7-year-old children with autism showed face memory impairments, children with milder social symptoms (those with PDD-NOS) did not show impairments (though see Serra et al., 2003). However, other studies have failed to find evidence of a relation in 9-year-old children (Hauck, Fein, Maltby, Waterhouse, \& Feinstein, 1998) or adults (Barton et al., 2004) with ASD. Possibly, social and communication skills are more closely correlated with face processing in the early development of ASD. Alternatively, face-processing disruptions may correlate more strongly with some 
social and communicative measures than others. In the present study, there were significant correlations with observed behaviors but no significant correlations with parent-reported symptoms (also see Klin et al., 1999). Examining correlations between ASD symptoms and face recognition across development with a range of measures is an important topic for future research.

What could account for slower face learning in ASD? Differences in the way faces are visually explored in toddlers with ASD (Jones et al., 2008) may disrupt learning about face stimuli. For example, a bias to attend to individual features of the face (Joseph \& Tanaka, 2003; Klin, Jones, Schultz, Volkmar, \& Cohen, 2002; Lahaie et al., 2006) might slow learning in toddlers with ASD, relative to other groups of toddlers who tend to process the face in a more holistic fashion. Sampling different features on each face presentation may decrease the retrieval cues present at each repetition, thus slowing habituation. Similarly, evidence from electrophysiological recordings suggests that the structural encoding of facial stimuli may be slower in 3- and 4year-olds with ASD (Webb, Dawson, Bernier, \& Panagiotides, 2006), raising the possibility that slowed encoding of structural features may in turn slow face learning over longer time scales.

In the present study, toddlers who did not have high symptoms of ASD did not differ in their time to habituate to faces or houses, either within or across diagnostic groups. However, the variables related to response to faces did differ between groups. Regression analyses revealed that looking time to houses significantly predicted looking time to faces in the $\mathrm{TD}_{\text {all }}$ group, as it did in the $\mathrm{ASD}_{\text {all }}$ group. This suggests that domain general stimulus-processing mechanisms contribute to individual differences in learning rates for social stimuli (see also Hauck et al., 1998). However, it is important to note that the present study found no evidence that these domain general learning mechanisms could account for slowed face learning or were impaired in ASD, as there were no differences between groups in time to habituate to houses.

Intriguingly, in the $\mathrm{DD}_{\text {all }}$ group habituation time to faces was not related to habituation time to houses. This may indicate that learning mechanisms are less consistent in the DD group, perhaps in part due to the heterogeneity of this diagnostic category. Alternatively, it may be that the DD group exhibited larger fluctuations within and between sessions than the ASD or TD groups, which would reduce the correlation between habituation time to faces and habituation time to houses. Finally, there may be reduced power to detect an effect in the DD group, as there were fewer children in this group than in the ASD or TD groups. Further work will be required to differentiate these possibilities.

Unlike the $\mathrm{ASD}_{\text {all }}$ group, verbal DQ and ADOS social and communication total score did not predict habituation time to faces in the $\mathrm{TD}_{\text {all }}$ group or in the $\mathrm{DD}_{\text {all }}$ group. As $\mathrm{ADOS}$ scores were very low in the typical group (range $=0$ to 8 in the $\mathrm{TD}_{\text {all }}$ group and 0 to 13 in the $\mathrm{DD}_{\text {all }}$ group), range restriction may have masked any relation with face learning. However, this was less likely to be the case for verbal DQ (range $=86$ to 128 in the $\mathrm{TD}_{\text {all }}$ group, and 48 to 108 in the $\mathrm{DD}_{\text {all }}$ group). Indeed, a range of evidence suggests that face learning and social and communication skills are not closely linked in individuals who are not on the autism spectrum (though see Hauck et al., 1998). For example, individuals with developmental prosopagnosia (clinical difficulties with face recognition) can develop relatively typical language skills (Duchaine, 2000); although they may suffer significant psychosocial difficulties as a result of face-recognition problems (Yardley, McDermott, Pisarsky, Duchaine, \& Nakayama, 2008). Potentially, face-processing ability and social, verbal, or communicative skill only correlate when both abilities are impaired. Longitudinal follow-up may illuminate the nature of the relation between face-processing impairment and social and communicative skills in ASD. 


\section{Interpreting Null Preferences in Toddlers}

Results from the test procedure indicated that for children who participated in all four test sessions, there were no differences in novelty preferences between different diagnostic groups, or between different stimulus types. The lack of a significant consistent novelty preference in the typically developing group is unexpected and clearly makes it difficult to interpret the absence of novelty preferences in the ASD group. Thus, the present findings do not enable us to evaluate whether toddlers with ASD show impairments in face recognition relative to toddlers with DD or typical development.

This observation raises a question about the validity of our paradigm: Does the absence of a novelty preference in the TD group mean that learning did not occur during the habituation phase? A decrease in looking to a stimulus over time can occur either because (a) toddlers learn something about the stimulus, or (b) because they become bored of looking at a screen or sitting still in a chair. Finding a novelty preference during the "dishabituaton" phase or demonstrating category level habituation enables the second explanation to be ruled out. In the present dataset, each habituation session contained two procedures (one with a face stimulus and one with a house stimulus), run sequentially. By comparing the peak look to the first stimulus (e.g., face) with the peak look to the second stimulus (e.g., house), we can examine whether toddlers dishabituated to the second "out-of-category" stimulus (a strategy also employed by Brian, Landry, Szatmari, Niccols, \& Bryson, 2003). In our study, peak looks to the first and second stimuli did not significantly differ for any comparison (all $F<1, p\rangle .4$ ), indicating that toddlers increased looking to an out-of-category stimulus. Thus, we can conclude that habituation was category specific. Of note, many studies that have observed dishabituation in sequential paradigms used out-of-category "novel" stimuli (e.g., Bornstein \& Benasich, 1986; Laucht, Becker, \& Schmidt, 2006; Mayes, Bornstein, Chawarska, \& Grainger, 1995). Since the conclusions we draw, slowed habituation to faces versus houses, concern category-level effects, we believe that the present interpretation of the habituation data is valid.

Why, in general, did we fail to observe a within-category novelty preference? Several possibilities exist. First, the use of a reorienting stimulus may have interfered with the typical learning process. However, many other studies have employed a reorienting stimulus and still observed significant novelty preferences (e.g., Bornstein \& Benasich, 1986; Lewkowicz, 2004; Saffran et al., 2007; Soska \& Johnson, 2008). Further, in the present study typically developing toddlers showed a significant novelty preference after a 5-minute but not a 5-second delay for the house stimulus. Since the exact same habituation procedure was used in each condition, it seems improbable that learning would have occurred in one condition but not the other.

Second, the present study may have been underpowered to detect novelty preferences in this paradigm. Consider the contrast between the present data and those presented by Chawarska and Shic (2009); referred to as "C\&S." C\&S explored novelty preferences for face stimuli in 2- to 4-year-old children with ASD or TD. In C\&S, the TD group $(n=30)$ showed a novelty preference of $0.56(S D=0.07 ; p<.001)$ after a 12 -second delay for a familiarized face simultaneously paired with a novel face. In the present Face Short Condition, the TD group $(n=22)$ showed a novelty preference of $0.55(S D=0.22 ; p=.29, \mathrm{~ns})$, and the TD-SIB group $(n=29)$ showed a novelty preference of $0.58(S D=0.2 ; p=.07)$. Notably, the mean novelty preferences observed were very similar in the present study and in C\&S. However, the variability within the TD and TD-SIB groups was much greater, reducing our power to detect an effect. When we tested the novelty preference of all typically developing children in the sample $\left(\mathrm{TD}_{\text {all }} ; n=44\right)$, the resulting novelty preference of $0.56(S D=0.21)$ was significant, $t$ $(47)=2.1, p=.04$. Indeed, using this approach, typically developing children showed a significant or nearly significant preference in all four conditions, House Short: $t(44)=2.1, p$ $=.043$; House Long: $t(44)=3.07, p=.004$; Face Long condition: $t(46)=1.73, p=.091$, whilst 
the ASDall group did not show a significant novelty preference in any condition ( $t \mathrm{~s}<1.8, p$ $>$.05).

Why would greater variability of looking during the test phase be observed in our study? Possibly, the "infant-controlled" habituation paradigm we employed leads to an increase in variability during the test session in comparison to a fixed familiarization period. Alternatively, sequentially presented test stimuli with free viewing may leave more room for variability in looking behavior than simultaneously presented test stimuli with a fixed duration of presentation (e.g., Caron, Caron, Minichiello, Weiss, \& Friedman, 1977) and may be less appropriate for assessing novelty preferences in this age range.

Lastly, it is important to recognize that a wealth of literature indicates that the lack of significant novelty preferences on an immediate test does not indicate that infants fail to learn about the stimulus during a habituation period (for review see Pascalis \& de Haan, 1998). For example, null preferences have been observed in infants and adults who, in a subsequent test session, demonstrate memory for the exact same stimulus using other means (Gross, Hayne, Herbert, \& Sowerby, 2002; Richmond, Colombo, \& Hayne, 2007; Snyder, Blank, \& Marsolek, 2008), or through a novelty preference (e.g., Bahrick \& Pickens, 1995; Courage \& Howe, 1998). Thus, whilst the absence of a novelty preference makes it more difficult to conclude that learning has occurred, it does not prove the converse - that learning has not occurred.

In summary, it appears that toddlers habituated to at least the category of stimulus with which they were presented, and that this habituation process to faces took longer in toddlers with severe symptoms of ASD. However, we cannot draw conclusions about whether they recognized the specific face exemplar because of the limitations of the novelty preference method that we employed.

\section{Could Face-Processing Impairments be an Endophenotype of ASD?}

Typically developing children with siblings with ASD tended to take longer to habituate to a face than typically developing children without a sibling with ASD. Regression analyses indicated that this effect cannot be attributed to variation in age, nonverbal DQ, or stimulus learning rate. This may indicate an association between familial risk factors for ASD, and relatively slowed face learning. Indeed, a range of cognitive, social, and communicative deficits have been observed in toddler or infant siblings of children with ASD (e.g., Cassel et al., 2007; Goldberg et al., 2005; Stone, McMahon, Yoder, \& Walden, 2007; Toth, Dawson, Meltzoff, Greenson, \& Fein, 2007; Yirmiya et al., 2006; Yirmiya, Gamliel, Shaked, \& Sigman, 2007), including abnormal patterns of visual attention to faces (Merin et al., 2007). Further, disrupted behavioral and neural face processing have been observed in the parents of children with ASD (Dawson et al., 2005). These observations are consistent with the proposition that slowed face processing is an endophenotype of ASD. However, the effects observed in the present study were very small and were only statistically significant in direct comparisons within the TD group. Thus, replication of this pattern would be required to draw strong conclusions about face learning in toddlers with siblings with ASD.

Apparently contrary to this hypothesis is the observation that sibling status did not appear to influence face learning in the group of children with general developmental delays. However, examination of Figure 1 indicates that both the DD and the DD-SIB groups habituated to the face stimulus more slowly than the TD group. Possibly, developmental delay also produces a decrement in face learning, masking any effect of sibling status in this group. Notably, Chawarska and Volkmar (2007) found that toddlers with general developmental delay also showed a deficit in face recognition relative to a group with typical development. Thus, a mild slowing of face learning may be a familial risk factor for ASD that is observed early in 
development, but may also be observed in toddlers with more general developmental delays. Further work is required to establish the sensitivity and specificity of this pattern.

\section{The Development of Face Processing in ASD}

In the present study, a relative slowing in face processing was observed in 18- to 30-monthold toddlers with ASD. Thus, differences in face processing can be observed at the earliest age ASD can currently be reliably diagnosed. A slight slowing was also observed in toddler siblings of children with ASD. Together, these observations suggest that face processing may be a specific area of disruption in ASD and the broader autism phenotype. Notably in the ASD group, the lack of correlation between slowed face processing and non-verbal DQ, and the typical speed of house processing, suggests that slowed face processing may not be explained by domain-general perceptual processing differences. Further, the relation between faceprocessing speed and social and communication symptoms in toddlers with ASD suggest either that the speed of face processing either contributes to impairments in other social and communicative domains, or that social and communicative impairments compound difficulties with face processing. Converging evidence suggests that a relation between social and communication skills and face-processing skills exists early in the development of children with ASD. Resolving the direction of this effect will be crucial in evaluating the necessity of early intervention for face-processing difficulties in ASD.

\section{Limitations and Future Directions}

One limitation of the present study is inherent in studying very young children: While the majority of children with ASD can be identified during toddlerhood, for some children, symptoms only become diagnostic later in development. Notably, the presence of children with undiagnosed ASD in the TD or DD samples would only serve to decrease the likelihood of observing differences in habituation times between the ASD group and other children. However, the question of late symptom emergence raises the intriguing possibility that the slightly slowed habituation to faces observed in the DD group (or in the TD-SIB or DD-SIB groups) was driven by a few children who will later receive an ASD diagnosis. A subset of the ASD and DD groups in the present study are currently being followed longitudinally, which will enable us to examine diagnostic stability and the relation of early face processing to later social abilities.

A further limitation of the present study was that toddlers did not show a consistent pattern of novelty preferences. This makes it more difficult to ascertain whether learning was stimulus specific, categorical, or related to aspects of the testing environment. However, alternative measures of memory suitable for this population are scarce. For example, many measures of memory used with typically developing toddlers rely on social learning (e.g., deferred imitation) or rule learning (e.g., delayed non-match to sample), involving other known domains of impairment in children with ASD. We may need to devise new measures of memory for toddlers who have significant developmental delays in order to accurately assess cognition across a wide spectrum of functioning.

Experimenters who coded the looking behavior of the child during the experiment were also not blind to their diagnostic status. Given that this project was part of a larger study and that many children had behaviors that were indicative of early autism or delay, it was not possible to keep experimenters blind to diagnostic status. Further, it is unlikely that this knowledge influenced the accuracy of the results because (a) the interrater reliability was high for all coded files, and (b) whilst experimenters were aware that children did or did not have ASD, they did not have access to ADOS scores (and hence the difference between the ASD-HS and ASD-LS groups) nor other influential measurement information, such as Mullen scores or language tests. 
Finally, the present study does not enable us to examine whether slowed face learning results from decreased experience with faces, or from a primary visual system abnormality present very early in development. Prospective studies of infants at risk for developing ASD (such as infants with siblings with ASD) may enable this possibility.

\section{Conclusion}

Slowed face learning was observed in a group of toddlers with ASD and slower face learning was correlated with lower verbal DQ and higher levels of ASD symptoms in the social and communicative domains. Typically developing toddlers with siblings with ASD also showed slower face learning than toddlers without siblings with ASD. We conclude that face-learning disruptions can be observed at the earliest age ASD is presently diagnosed and that they are related to the social and communication impairments that are central to the condition. Further, mild disruptions in face learning may be a feature of the "broader phenotype" of ASD and apparent very early in development.

\section{Acknowledgments}

This research was funded by a program project grant from the NIMH Studies to Advance Autism Research and Treatment (U54MH066399). We gratefully acknowledge the contributions of these funding sources, the Clinical and Statistical Cores of this project, and the individuals who participated in this study.

\section{REFERENCES}

Adolphs R. Neural systems for recognizing emotion. Current Opinion in Neurobiology 2002;12(2):169_ 177. [PubMed: 12015233]

Adolphs R, Spezio ML, Parlier M, Piven J. Distinct face-processing strategies in parents of autistic children. Current Biology 2008;18(14):1090-1093. [PubMed: 18635351]

Adolphs R, Tranel D. Amygdala damage impairs emotion recognition from scenes only when they contain facial expressions. Neuropsychologia 2003;41(10):1281-1289. [PubMed: 12757901]

Akshoomoff N. Use of the Mullen scales of early learning for the assessment of young children with autism spectrum disorders. Child Neuropsychology 2006;12:269-277. [PubMed: 16911972]

American Psychiatric Association. Diagnostic and statistical manual of mental disorders. 4th ed.. Author; Washington, DC: 1994.

Bahrick L, Pickens CL. Infant memory for object motion across a period of three months: Implications for a four-phase attention function. Journal of Experimental Child Psychology 1995;59:343-371. [PubMed: 7622984]

Bailey A, Le Couteur A, Gottesman I, Bolton P, Simonoff E, Yuzda E, et al. Autism as a strongly genetic disorder: Evidence from a British twin study. Psychological Medicine 1995;25:63-77. [PubMed: 7792363]

Baldwin DA. Infants' ability to consult the speaker for clues to word reference. Journal of Child Language 1993;20(2):395-418. [PubMed: 8376476]

Barton JJS, Cherkasova MV, Hefter R, Cox TA, O'Connor M, Manoach DS. Are patients with social developmental disorders prosopagnosic? Perceptual heterogeneity in the Asperger and socioemotional processing disorders. Brain 2004;127(8):1706. [PubMed: 15215211]

Behrmann M, Thomas C, Humphreys K. Seeing it differently: Visual processing in autism. Trends in Cognitive Sciences 2006;10(6):258-264. [PubMed: 16713326]

Bernabei P, Camaigni L, Levi G. An evaluation of early development in children with autism and pervasive developmental disorders from home movies: Preliminary findings. Autism 1998;2(3):243258.

Bornstein MH, Benasich AA. Infant habituation: Assessments of individual differences and short-term reliability at five months. Child Development 1986;57(1):87-99. [PubMed: 3948596]

Bornstein MH, Sigman MD. Continuity in mental development from infancy. Cognitive Development in Infancy: A Reader 1987;57:251-274. 
Boucher J, Lewis V, Collis G. Familiar face and voice matching and recognition in children with autism. Journal of Child Psychology and Psychiatry 1998;39(2):171-181. [PubMed: 9669230]

Brian JA, Landry R, Szatmari P, Niccols A, Bryson S. Habituation in high-risk infants: Reliability and patterns of responding. Infant and Child Development 2003;12:387-394.

Brooks R, Meltzoff AN. The importance of eyes: How infants interpret adult looking behavior. Developmental Psychology 2002;38(6):958-966. [PubMed: 12428707]

Brooks R, Meltzoff AN. The development of gaze following and its relation to language. Developmental Science 2005;8(6):535-543. [PubMed: 16246245]

Caron AJ, Caron RF, Minichiello MD, Weiss SJ, Friedman SL. Constraints on the use of the familiarization-novelty method in the assessment of infant discrimination. Child Development 1977;48:747-762.

Casasola M, Bhagwat J. Do novel words facilitate 18-month-olds' spatial categorization? Child Development 2007;78(6):1818-1829. [PubMed: 17988323]

Cassel TD, Messinger DS, Ibanez LV, Haltigan JD, Acosta SI, Buchman AC. Early social and emotional communication in the infant siblings of children with autism spectrum disorders: An examination of the broad phenotype. Journal of Autism and Developmental Disorders 2007;37(1):122-132. [PubMed: 17186367]

Chawarska K, Shic F. Looking but not seeing: Atypical visual scanning and recognition of faces in 2 and 4-year-old children with autism spectrum disorder. Journal of Autism and Developmental Disorders 2009;39

Chawarska K, Volkmar F. Impairments in monkey and human face recognition in 2-year-old toddlers with Autism Spectrum Disorder and Developmental Delay. Developmental Science 2007;10:266279. [PubMed: 17286849]

Cohen, LB.; Atkinson, DJ.; Chaput, HH. Habit X: A new program for obtaining and organizing data in infant perception and cognition studies. University of Texas; Austin: 2004.

Colombo, J.; Mitchell, DW. Individual differences in early visual attention: Fixation time and information processing. In: Colombo, J.; Fagen, J., editors. Individual differences in infancy: Reliability, stability, prediction. Lawrence Erlbaum Associates; Mahwah, NJ: 1990. p. 193-227.

Colombo J, Mitchell DW. Infant visual habituation. Neurobiology of Learning and Memory 2008;92:225-234. doi:10.1016/j.nlm.2008.06.002. [PubMed: 18620070]

Colombo J, Mitchell DW, Horowitz FD. Infant visual attention in the paired-comparison paradigm: Testretest and attention-performance relations. Child Development 1988;59(5):1198-1210. [PubMed: 3168636]

Colombo J, Mitchell DW, O'Brien M, Horowitz FD. The stability of visual habituation during the first year of life. Child Development 1987;58(2):474-487. [PubMed: 3829788]

Courage ML, Howe ML. The ebb and flow of infants' attentional preferences: Evidence of long-term recognition memory in 3-month-olds. Journal of Experimental Child Psychology 1998;70:26-53. [PubMed: 9679078]

Dawson G, Carver L, Meltzoff AN, Panagiotides H, McPartland J, Webb SJ. Neural correlates of face and object recognition in young children with autism spectrum disorder, developmental delay, and typical development. Child Development 2002;73(3):700-717. [PubMed: 12038546]

Dawson G, Webb S, McPartland J. Understanding the nature of face processing impairment in autism: Insights from behavioral and electrophysiological studies. Developmental Neuropsychology, Special Issue of Autism 2005;27(3):403-424.

Duchaine BC. Developmental prosopagnosia with normal configural processing. Neuroreport 2000;11 (1):79-83. [PubMed: 10683834]

Fagan J. Infant recognition memory: The effects of length of familiarization and type of discrimination task. Child Development 1974;45:351-356. [PubMed: 4837713]

Fagan J. Recognition memory and intelligence. Intelligence 1984;8:31-36.

Freitag CM. The genetics of autistic disorders and its clinical relevance: A review of the literature. Molecular Psychiatry 2007;12:2-22. [PubMed: 17033636]

Goldberg WA, Jarvis KL, Osann K, Laulhere TM, Straub C, Thomas E, et al. Brief report: Early social communication behaviors in the younger siblings of children with autism. Journal of Autism and Developmental Disorders 2005;35:657-664. [PubMed: 16167088] 
Gottesman II, Gould TD. The endophenotype concept in psychiatry: Etymology and strategic intentions. American Journal of Psychiatry 2003;160:636-645. [PubMed: 12668349]

Grelotti D, Gauthier I, Schultz R. Social interest and the development of cortical face specialization; What autism teaches us about face processing. Developmental Psychobiology 2002;40:213-225. [PubMed: 11891634]

Gross J, Hayne H, Herbert J, Sowerby P. Measuring infant memory: Does the ruler matter? Developmental Psychobiology 2002;40(2):183-192. [PubMed: 11857332]

Gupta AR, State MW. Recent advances in the genetics of autism. Biological Psychiatry 2007;61(4):429_ 437. [PubMed: 16996486]

Hauck M, Fein D, Maltby N, Waterhouse L, Feinstein C. Memory for faces in children with autism. Child Neuropsychology 1998;4(3):187-198.

Hobson RP. Methodological issues for experiments on autistic individuals' perception and understanding of emotion. Journal of Child Psychology and Psychiatry 1991;32(7):1135-1158. [PubMed: 1787140]

Hollich G, Hirsh-Pasek K, Golinkoff RM. II. The emergentist coalition model. Monographs of the Society for Research in Child Development 2000;65(3):17-29.

Houston-Price C, Plunkett K, Duffy H. The use of social and salience cues in early word learning. Journal of Experimental Child Psychology 2006;95(1):27-55. [PubMed: 16677668]

Ibanez LV, Messinger DS, Newell L, Lambert B, Sheskin M. Visual disengagement in the infant siblings of children with an autism spectrum disorder (ASD). Autism 2008;12(5):473. [PubMed: 18805943]

Jarrold C, Brock J. To match or not to match? Methodological issues in autism-related research. Journal of Autism and Developmental Disorders 2004;34(1):81-86. [PubMed: 15098961]

Jemel B, Mottron L, Dawson M. Impaired face processing in autism: Fact or artifact? Journal of Autism and Developmental Disorders 2006;36(1):91-106. [PubMed: 16477517]

Jones EJH, Pascalis O, Eacott MJ, Herbert JS. Visual recognition memory across contexts. Developmental Science. in press.

Jones W, Carr K, Klin A. Absence of preferential looking to the eyes of approaching adults predicts level of social disability in 2-year-old toddlers with autism spectrum disorder. Archives of General Psychiatry 2008;65(8):946. [PubMed: 18678799]

Joseph RM, Tanaka J. Holistic and part-based face recognition in children with autism. Journal of Child Psychology and Psychiatry, and Allied Disciplines 2003;44(4):529-542.

Klin A, Jones W, Schultz R, Volkmar F, Cohen D. Visual fixation patterns during viewing of naturalistic social situations as predictors of social competence in individuals with autism. Archives of General Psychiatry 2002;59:809-816. [PubMed: 12215080]

Klin A, Sparrow SS, de Bildt A, Cicchetti DV, Cohen DJ, Volkmar FR. A normed study of face recognition in autism and related disorders. Journal of Autism and Developmental Disorders 1999;29:499-508. [PubMed: 10638462]

Kuhl P, Tsao F, Liu H. Foreign-language experience in infancy: Effects of short-term exposure and social interaction on phonetic learning. Proceeding of the National Academy of Sciences 2003;100(15): 9096-9101.

Lahaie A, Mottron L, Arguin M, Berthiaume C, Jemel B, Saumier D. Face perception in high-functioning autistic adults: Evidence for superior processing of face parts, not a configural face-processing deficit. Neuropsychology 2006;20:30-41. [PubMed: 16460220]

Laucht M, Becker K, Schmidt MH. Visual exploratory behavior in infancy and novelty seeking in adolescence: Two developmentally specific phenotypes of DRD4? Journal of Child Psychology and Psychiatry 2006;47(11):1143-1151. [PubMed: 17076753]

Leboyer M, Bellivier F, Nosten-Bertrand M, Jouvent R, Pauls D, Mallet J. Psychiatric genetics: Search for phenotypes. Trends in Neuroscience 1998;21:102-105.

Lewkowicz DJ. Perception of serial order in infants. Developmental Science 2004;7(2):175-184. [PubMed: 15320377]

Lord C, Risi S, DiLavore PS, Shulman C, Thurm A, Pickles A. Autism from 2 to 9 years of age. Archives of General Psychiatry 2006;63:694-701. [PubMed: 16754843]

Lord, C.; Rutter, M.; DiLavore, PC.; Risi, S. Autism diagnostic observation schedule - WPS (ADOSWPS). Western Psychological Services; Los Angeles, CA: 1999. 
Lord C, Rutter M, Le Couteur A. Autism diagnostic interview - revised: A revised version of a diagnostic interview for caregivers of individuals with possible pervasive developmental disorders. Journal of Autism and Developmental Disorders 1994;24:659-685. [PubMed: 7814313]

Maestro S, Muratori F, Barbieri F, Casella C, Cattaneo V, Cavallaro MC, et al. Early behavioral development in autistic children: The first 2 years of life through home movies. Psychopathology 2002;34(3):147-152. [PubMed: 11316961]

Maestro S, Muratori F, Cesari A, Cavallaro MC, Paziente A, Pecini C, et al. Course of autism signs in the first year of life. Psychopathology 2005;38(1):26-31. [PubMed: 15731570]

Mars AE, Mauk JE, Dowrick PW. Symptoms of pervasive developmental disorders as observed in prediagnostic home videos of infants and toddlers. Journal of Pediatrics 1998;132:1-5. [PubMed: 9469990]

Mayes LC, Bornstein MH, Chawarska K, Grainger RH. Information processing and developmental assessments in 3-month-old infants exposed prenatally to cocaine. Pediatrics 1995;95:539-545. [PubMed: 7700755]

McCall RB, Carriger MS. A meta-analysis of infant habituation and recognition memory performance as predictors of later IQ. Child Development 1993;64:57-79. [PubMed: 8436038]

McCleery JP, Allman E, Carver LJ, Dobkins KR. Abnormal magnocellular pathway visual processing in infants at risk for autism. Biological Psychiatry 2007;62(9):1007-1014. [PubMed: 17531206]

McGurk H, McDonald J. Hearing and seeing voices. Nature 1976;264:746-748. [PubMed: 1012311]

Merin N, Young G, Ozonoff S, Rogers S. Visual fixation patterns during reciprocal social interaction distinguish a subgroup of 6-month-old infants at-risk for autism from comparison infants. Journal of Autism and Developmental Disorders 2007;37(1):108-121. [PubMed: 17191096]

Moore C, Angelopoulos M, Bennett P. Word learning in the context of referential and salience cues. Developmental Psychology 1999;35(1):60-68. [PubMed: 9923464]

Mullen, EM. Mullen Scales of Early Learning. Western Psychological Services; Los Angeles, CA: 1997.

Munson J, Dawson G, Sterling L, Beauchaine T, Zhou A, Koehler E, et al. Evidence for latent classes of IQ in young children with autism spectrum disorder. American Journal on Mental Retardation 2008;113:439-452. [PubMed: 19127655]

Osterling J, Dawson G. Early recognition of children with autism: A study of first birthday home videotapes. Journal of Autism and Developmental Disorders 1994;24:247-257. [PubMed: 8050980]

Osterling JA, Dawson G, Munson JA. Early recognition of 1-year-old infants with autism spectrum disorder versus mental retardation. Developmental Psychopathology 2002;14(2):239-251.

Pascalis, O.; de Haan, M. Recognition memory and novelty preference: What model?. In: Hayne, H.; Fagen, J., editors. Progress in infancy research. Vol. Vol. 2. Lawrence Erlbaum Associates; Mahwah, NJ: 1998. p. 95-121.

Pascalis O, de Haan M, Nelson CA, de Schonen S. Long-term recognition memory for faces assessed by visual paired comparison in 3- and 6-month-old infants. Journal of Experimental Psychology: Learning, Memory, \& Cognition 1998;24(1):249-260.

Rankin CH, Abrams T, Barry RJ, Bhatnagar S, Clayton DF, Colombo J, et al. Habituation revisited: An updated and revised description of the behavioral characteristics of habituation. Neurobiology of Learning and Memory 2009;92:135-138. [PubMed: 18854219]

Richmond J, Colombo J, Hayne H. Interpreting visual preferences in the visual paired-comparison task. Journal of Experimental Psychology: Learning, Memory, \& Cognition 2007;33(5):823-831.

Rose SA, Feldman JF, Jankowski JJ. The structure of infant cognition at 1 year. Intelligence 2004;33(3): 231-250.

Rose SA, Feldman JF, Jankowski JJ. Recall memory in the first three years of life: A longitudinal study of preterm and term children. Developmental Medicine and Child Neurology 2005;47:653-659. [PubMed: 16174307]

Rose SA, Feldman JF, Wallace IF. Individual differences in infants' information processing: Reliability, stability, and prediction. Child Development 1988;59(5):1177-1197. [PubMed: 3168635]

Rose SA, Feldman JF, Wallace IF. Infant information processing in relation to six-year cognitive outcomes. Child Development 1992;63(5):1126-1141. [PubMed: 1446544] 
Saffran JR, Pollak SD, Seibel SD, Shkolnik A. Dog is a dog is a dog: Infant rule learning is not specific to language. Cognition 2007;105(3):669-680. [PubMed: 17188676]

Sasson NJ. The development of face processing in autism. Journal of Autism and Developmental Disorders 2006;36:381-394. [PubMed: 16572261]

Scherf KS, Behrmann M, Minshew N, Luna B. Atypical development of face and greeble recognition in autism. Journal of Child Psychology and Psychiatry, and Allied Disciplines 2008;49(8):838-847.

Serra M, Althaus M, de Sonneville LMJ, Stant AD, Jackson AE, Minderaa RB. Face recognition in children with a pervasive developmental disorder not otherwise specified. Journal of Autism \& Developmental Disorders 2003;33(3):303-317. [PubMed: 12908833]

Skuse DH. Rethinking the nature of genetic vulnerability to autistic spectrum disorders. Trends in Genetics 2007;23(8):387-395. [PubMed: 17630015]

Slater, A.; Bremner, G. Infant development. Psychology Press; Hove, UK: 1989.

Snyder KA, Blank MP, Marsolek CJ. What form of memory underlies novelty preferences? Psychonomic Bulletin and Review 2008;15(2):315-321. [PubMed: 18488646]

Soska KC, Johnson SP. Development of three-dimensional object completion in infancy. Child Development 2008;79(5):1230-1236. [PubMed: 18826522]

Sparrow, S.; Balla, D.; Cicchetti, D. Vineland Adaptive Behavior Scales: Interview edition. American Guidance Service; Circle Pines, MN: 1984.

Steffenburg S, Gillberg C, Hellgren L, Andersson L, Gillberg I, Jakobsson G, et al. A twin study of autism in Denmark, Finland, Iceland, Norway, and Sweden. Journal of Child Psychology and Psychiatry and Allied Disciplines 1989;30:405-416.

Stone WL, McMahon CR, Yoder PJ, Walden TA. Early social-communicative and cognitive development of younger siblings of children with autism spectrum disorders. Archives of Pediatrics and Adolescent Medicine 2007;161(4):384. [PubMed: 17404136]

Strauss MS, Curtis LE. Infant perception of numerosity. Child Development 1981;52(4):1146-1152. [PubMed: 7318517]

Thompson LA, Fagan JF, Fulker DW. Longitudinal predictions of specific cognitive abilities from infant novelty preference. Child Development 1991;62(3):530-538. [PubMed: 1914623]

Tomasello M, Barton ME. Learning words in nonostensive contexts. Developmental Psychology 1994;30:639-650.

Toth K, Dawson G, Meltzoff AN, Greenson J, Fein D. Early social, imitation, play and language abilities of young non-autistic siblings of children with autism. Journal of Autism and Developmental Disorders 2007;37(1):145-157. [PubMed: 17216560]

van Kooten IAJ, Palmen SJMC, von Cappeln P, Steinbusch HWM, Korr H, Heinsen H, et al. Neurons in the fusiform gyrus are fewer and smaller in autism. Brain 2008;131(4):987. [PubMed: 18332073]

Webb SJ, Dawson G, Bernier R, Panagiotides H. ERP evidence of atypical face processing in young children with autism. Journal of Autism and Developmental Disorders 2006;36(7):881-890. [PubMed: 16897400]

Wellman HM, Phillips AT, Dunphy-Lelii S, LaLonde N. Infant social attention predicts preschool social cognition. Developmental Science 2004;7(3):283-288. [PubMed: 15595369]

Yardley L, McDermott L, Pisarski S, Duchaine B, Nakayama K. Psychosocial consequences of developmental prosopagnosia: A problem of recognition. Journal of Psychosomatic Research 2008;65:445-451. [PubMed: 18940375]

Yirmiya N, Gamliel I, Pilowsky T, Feldman R, Baron-Cohen S, Sigman M. The development of siblings of children with autism at 4 and 14 months: Social engagement, communication, and cognition. Journal of Child Psychology and Psychiatry 2006;47(5):511-523. [PubMed: 16671934]

Yirmiya N, Gamliel I, Shaked M, Sigman M. Cognitive and verbal abilities of 24-to 36-month-old siblings of children with autism. Journal of Autism and Developmental Disorders 2007;37(2):218-229. [PubMed: 16897384]

Zakian A, Malvy J, Desombre H, Roux S, Lenoir P. Early signs of autism and family films: A new study by informed evaluators and those unaware of the diagnosis. L'Encephale 2000;26(2):38-44.

Zimmerman, V.; Steiner, R.; Pond, R. Preschool Language Scale. 4th ed.. The Psychological Corporation; San Antonio, TX: 2002. 




Figure 1.

Examples of stimuli used in the habituation procedure. 


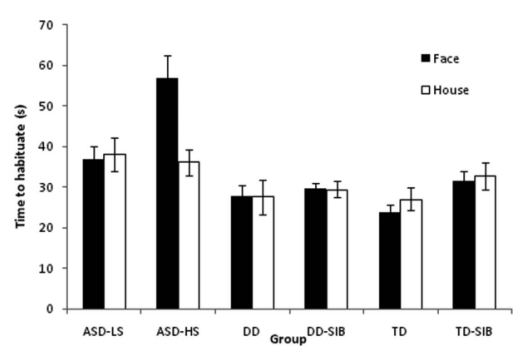

Figure 2.

Mean time to habituate (seconds) to faces and houses in the six groups of children; error bars represent \pm one standard error. 







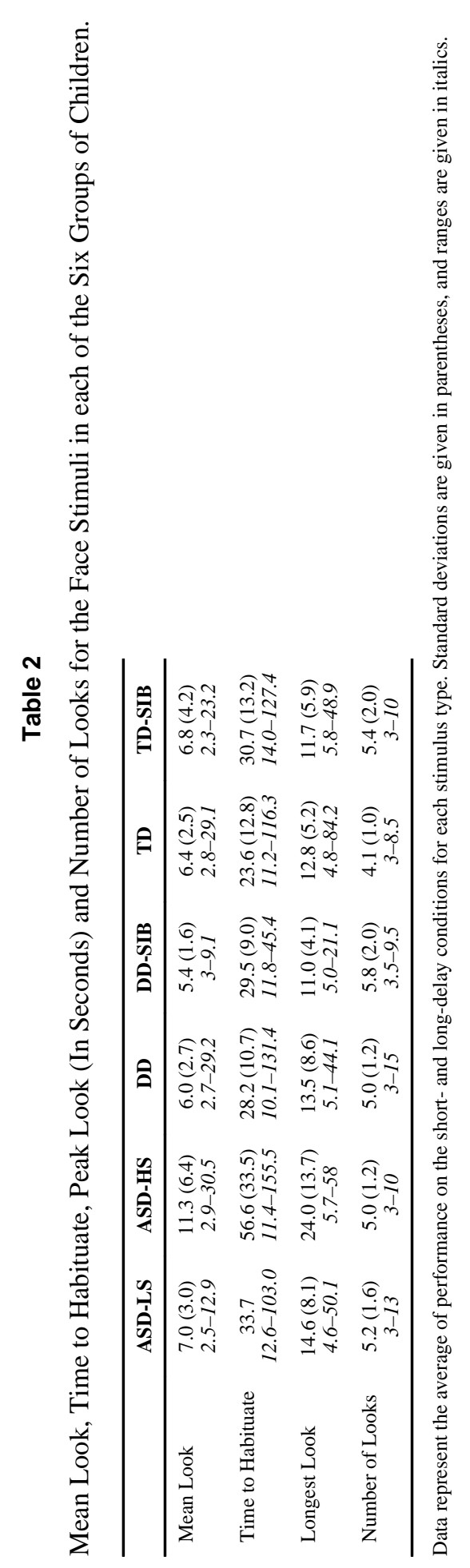

Child Neuropsychol. Author manuscript; available in PMC 2011 May 1. 




Child Neuropsychol. Author manuscript; available in PMC 2011 May 1. 




Child Neuropsychol. Author manuscript; available in PMC 2011 May 1. 